\title{
The Application of Histogram on Rain Detection in Video
}

\author{
Xudong Zhao， Peng Liu， Jiafeng Liu， Xianglong Tang \\ Department of Computer Science, Harbin Institute of Technology, China, 150001
}

\begin{abstract}
Rain, which is randomly distributed and falls at high speed, behaves complex in video and makes the work of rain detection hard. The improved histogram model is proposed for detection and removal of rain in video. The properties of this model, which are suitable for both stationary and dynamic scenes, are particularly discussed. An appropriate and concise K-means clustering algorithm is embedded in our methods, which remarkably reduces the complexity of the existing algorithm. The effectiveness of our methods is demonstrated using experimental results, which corroborates the significance of the intensity property for detection and removal of rain in video.
\end{abstract}

Keywords: Rain; Detection; Image Restoration; Image Processing; Video Enhancement

\section{Introduction}

Weather conditions can be broadly classified as steady (fog, mist and haze) or dynamic (rain, snow and hail) judging from the sizes of individual droplets [1]. Raindrops or snowflakes are randomly distributed and move all the time, which makes those methods used in steady weather conditions [2] lapse. Note that each raindrop covers only a small period in each frame of a video. When raindrops cover the background information, image inpainting [3] seems to be effective, in which only information of neighboring area can be used. Considering a raindrop is transparent [4] and not always covering the same pixel, it just hides background information. As a result, image restoration, rather than image inpainting, is the right topic on rain detection in video [5].

In the spatial domain, rain in video presents two remarkable properties as follows:

- Rain's transparency, which can refract the light from the reflection of the background, makes rain streak bright, even image the background.

- Video's continuity, which indicates that information of located pixels varies from rain to background at times.

Garg and Nayar investigated the physical properties of rain [1]. Raindrops of small size enlarge the drop density, which restrict the shape, velocity and the distribution. Based on the velocities and distribution of raindrops, Garg and Nayar successfully established the dynamic model of rain streak; moreover, in their photometry model of rain drops and streaks, they indicated that a drop stayed only a single frame over a pixel and the change of intensity along the rain streak were related to the background.

Considering global properties of rain streak recognition or rain detection such as physical and statistical characteristics, Narasimhan [6] developed a directional filter model in frequency space to detect

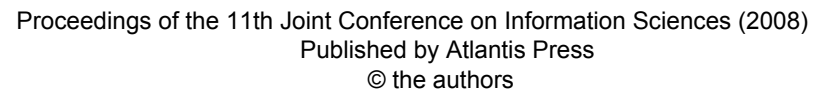


rain and snow streak. It works well when the velocity of raindrops or snowflakes is high, for image projections of raindrops or snow flakes present streaks in certain direction; however, it'll be of no effect because of the large size of snowflake and effect of wind.

These methods suggest the directional streak to be the first property for rain recognition, yet it is more effective when intensity property is applied to recognize rain and snow. Considering the background of high intensity covered by motion-blurred raindrops, even a human would have difficulty pointing out rain streaks, because of the low contrast.

Based on temporal and chromatic intensity properties, Zhang and his colleague [7] presented a rain detection algorithm, which could detect rain in both stationary and dynamic scenes, and handle conditions containing both light and heavy rain. However, their histogram model of intensity, which can be used only in stationary scenes, is established throughout the video; moreover, the chromatic property is empirical and depends strongly on the color distribution of the experimental frames.

Focusing on the intensity property of rain, this paper improves the histogram model, which is used in stationary scenes, both in temporal aspect and in spatial aspect. A new histogram model is established for pixels in dynamic scenes (here, only stand for the stationary scenes with moving objects). K-means clustering, which shows availability of classification, is applied to estimate the center of cluster for pixels. Experimental results show the efficiency and exactness of our methods.

\section{Temporal , Spatial and Dynamic Properties of Histogram Model}

Note that a pixel in a frame is not always covered by an image projection of a raindrop. It may also be an image projection of the background in a stationary scene or an image projection of the moving object in a dynamic scene. Pixels are concentrated on for modeling in this paper. Focusing on the stationary scene, we improve the histogram model in temporal and spatial properties. As to the dynamic scene, a new histogram model is developed in order to adapt the moving object.

\subsection{Temporal Property}

In stationary scenes, two different kinds of image projections appear in one pixel, one of which is derived from the background information, and the other one comes from the covering of the motionblurred raindrop. Note that only one peak of intensity histogram will appear in one pixel, if there is no rain throughout the video. Two main concentrative peaks of intensity histogram is no doubt to come forth, when there is no moving object in the scenes (Fig. 1(a)).

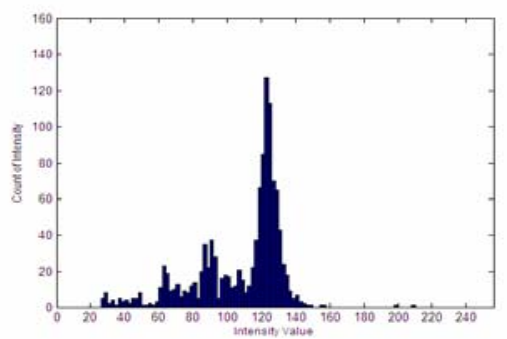

(a) Histogram of the whole video

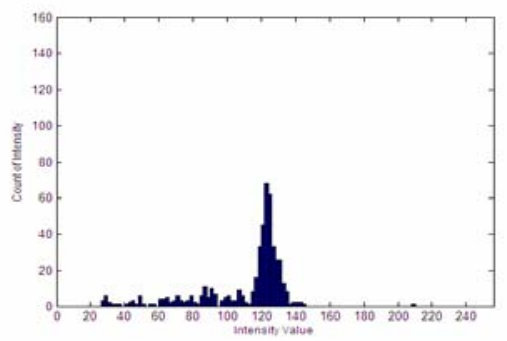

(b) Histogram of the half-length video

Fig. 1: Intensity histograms of one fixed pixel in video. (a) flowing bars of intensity for the whole video, where two peaks appear for the covering of raindrops and the stationary scene. (b) flowing bars of intensity for half length of




the video, whose figure and location of the peaks almost keep same with the bars for entire one.

Focused on one pixel during the video, the intensity histogram is relatively fixed, if there is not any moving object in stationary scenes. That means the existing established intensity histogram is almost fixed in figure and location when video of the stationary scenes without moving object continues. Fig. 1 illustrates the stability of one pixel's the intensity histogram during half of the video and the whole one. Based on the intuitionistic features obtained in Fig. 1, two obvious characters can be given to be the temporal properties as follows:

- As to a fixed pixel in video, two peaks, which stand for raindrops and the background, is no doubt to appear in its intensity histogram, if the scene is stationary and without any moving object.

- As to the above-mentioned intensity histogram, the locations of its two peaks are impossibly changed, no matter it is derived from the entire video or not.

On the whole, the intensity histogram of foregoing parts in video, instead of the entire video, is absolutely sufficient for establishing the rain detection model.

\subsection{Spatial Property}

In stationary scenes, there are some similarities and certain difference between the intensity histograms of two different pixels. Each of the intensity histogram has two peaks; moreover, the peak location which stands for the covering of raindrops is likely the same. However, peak location which stands for the background is obviously different. Pixels from different regions of the background appear different peaks.
The above-mentioned main difference is illustrated in Fig. 2, which reveals the importance of intensity property for rain recognition and dependence of the intensity histogram model on the intensity of background. Much higher peak value of intensity from the background than that from the covering of raindrops appears in Fig. 2(b), which explains the fact that recognition of raindrops covering background with high intensity is exceedingly difficult.



(a) Histogram of low intensity background



(b) Histogram of high intensity background

Fig. 2: Intensity histograms of two different pixels with different intensity in stationary scenes. (a) flowing bars of intensity for low intensity background, where the peak location of the background is lower than that of the covering of raindrops. (b) flowing bars of intensity for high intensity background, where the peak location of the background is higher than that of the covering of raindrops.

On the other hand, the intensity histograms of neighboring pixels appear almost same. Fig. 3 generally indicates this situation, compared with Fig. 2(a). Note that two neighboring pixels have almost the same histogram. This phenomenon is

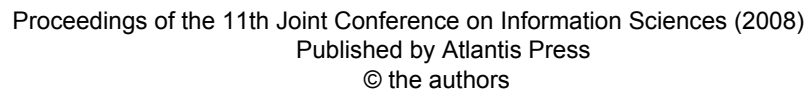


derived from the same intensity of the neighboring pixels and from the fact that raindrops have almost the same intensity.



Fig. 3: Histogram of the neighboring pixel. It is a histogram with low intensity background, and has almost the same peak locations with the histogram of Fig. 2(a).

Anyhow, similarities and difference are both well exhibited in spatial property. The neighboring pixels have the similar histogram models throughout the video, which can be applied to combine histograms with similarity in neighboring area and form a final histogram. Pixels with different intensity background appear different intensity histograms, which may help to segmentation; moreover, fact that pixels with high intensity background are ineffective in rain detection is vividly interpreted.

\subsection{Histogram Model for Dynamic Scenes}

Pixels in dynamic scenes, which present the image projections of background, are mainly covered by raindrops or by moving objects. Theoretically speaking, three peaks will appear in intensity histogram of one pixel, two of which stand for the background information and covering of raindrops. The third intending peak will stand for the moving objects. However, considering the difference in intensity of moving objects, the appearance of histogram may be complex. Pixels covered with moving objects of contrary intensity (compared with the intensity of the background) appear three peaks in their inten- sity histograms, whereas pixels covered with moving objects of similar intensity reserve only two peaks without much change. Compared with Fig. 2(a), the difference between the reserved two-peak histogram and the new three-peak histogram is distinctly illustrated in Fig. 4.

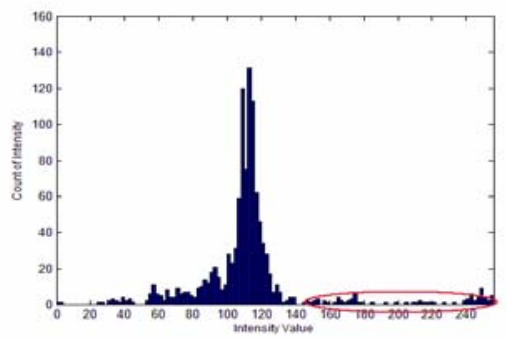

Fig. 4: Histogram of the three-peak. Considering contrary intensity of moving objects (e.g. the intensity of background stays low, while the intensity of moving objects appears high), three peaks should be undoubtedly confirmed before training. Note that the bars circled out stand for the moving objects of high intensity.

Considering the difference in distribution between raindrops and moving objects, temporal property is not applicable to the dynamic scenes. Although both the individual raindrops and moving objects are distributed randomly in 3D space, the distribution of rain, which is usually assumed to be uniform, remains constant over time by its statistical properties [8]. As a result, the training on histogram for dynamic scenes should be carried through during the video.

\section{K-means Clustering Algorithm for Rain Detection}

The intensity histogram of each pixel in video presents to be the Mixture Gauss Model. Considering that K-means Clustering is an effective algorithm for Mixture Gauss Distribution, it can be used to confirm the locations of cluster centers which stand for raindrops, background, and even the moving objects. In order to accelerate the processing rate of rain de-

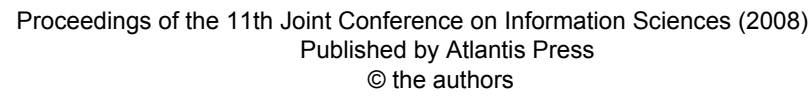


tection in dynamic scenes, concise twopeak histogram model than three-peak one is established.

\subsection{Training K-means Clustering for Stationary Scenes}

In stationary scenes, the intensity of each fixed pixel over the entire video is stored to compute the histogram. Then The obtained histograms are trained with $\mathrm{K}$ means clustering, where $K$ equals 2 . As to each histogram, two cluster centers $\omega_{b}$ for background and $\omega_{r}$ for raindrops are initialized to be the minimum and maximum of the intensity. The Euclid distance $d$ standing for the distance between the intensity $I$ in pixel $p$ and the cluster center $\omega$ is computed as the following equation,

$$
d\left(I_{p}, \omega\right)=\left|I_{p}-\omega\right|
$$

Pixel $p$ in frame $n$ is divided in raindrop cluster, if $d\left(I_{p}, \omega_{r}\right)$ is smaller than $d\left(I_{p}, \omega_{b}\right)$; otherwise it is divided in background cluster. After that, the center of cluster $C$ is updated as follows:

$$
\omega(n+1)=\frac{1}{|C(n)|} \sum_{I_{p} \in C(n)} I_{p} .
$$

K-means Clustering continues until the cluster centers converge [7]. Considering the difference for recognition of raindrops between lower intensity background and higher one, we believe that higher intensity background appears, if distance between the higher $\omega$ and the maximum of the intensity is within the limit $\theta_{l}$. In that case, the lower $\omega$ stands for $\omega_{b}$ and the higher one stands for $\omega_{r}$. The contrary case also works.

When cluster centers are confirmed, pixel $p$ in each frame can be considered to be the background or rain. If temporal property of histogram model is taken into account, the obtained cluster centers can be applied to further frames in video.

\subsection{Improved Histogram Model for Dynamic Scenes}

In dynamic scenes, theoretically moving objects will form the third peak in histogram. However, the three-peak histogram model appears complex, for the intensity of moving objects differs at times. Considering that the moving objects are randomly distributed, the temporal property is no longer fit for dynamic scenes. So we concentrate on improving the histogram model of stationary scenes.

Parameter estimation is usually effective on Gauss Distribution. By estimating the standard deviation $\sigma$, the boundary on distribution of raindrops can be easily obtained. However, each distribution in each histogram of each pixel is to be computed, which enlarges the volume of computation on standard deviation $\sigma$. A concise method, which estimates relative lowest sum of each intensity location in different histograms throughout the similar neighboring area (with a global point of view), is applied to obtain the boundary on distribution of raindrops from $\theta$. to $\theta_{+}$, whereas the intensity distribution of raindrops is relatively fixed.

Once the boundary is obtained, there is no need to calculate the Euclid distance $d$ again for classification on intensity of the fixed pixel in each frame. Instead, the boundary will work. If the intensity in the frame is within $\left(\theta_{-}, \theta_{+}\right)$, then the pixel in the frame is covered with rain. In order to remove the effect of rain, revisory $\omega_{b}$ ' is applied to replace the pixel's intensity.

\section{Experimental Results}

In our experiment, we used JVC GR-HD1 camera to take a video with different intensity of background, including moving objects. The former frames are of station-




ary scenes, while the latter frames contain moving objects. Matlab 7.0 is applied to store the data, simulate the clustering and calculate the boundary. Main parameters are estimated and filled in the following table,

\begin{tabular}{|c|c|c|c|c|c|c|}
\hline \multicolumn{2}{|c|}{$\omega_{b}$} & \multicolumn{2}{|c|}{$\omega_{r}$} & \multirow{2}{*}{$\theta_{l}$} & \multirow{2}{*}{$\theta_{-}$} & $\theta_{+}$ \\
\cline { 1 - 4 } $\min$ & $\max$ & $\min$ & $\max$ & & & \\
\hline 0 & 255 & 102 & 184 & 71 & 139 & 160 \\
\hline
\end{tabular}

Table 1: Parameters on cluster centers and on detection boundary. The values above, which are in uint8 form (from 0 to 255), stand for training values of intensity.

Based on the established histogram model and the algorithms of K-Means Clustering, rains in video are well detected and substituted by background information. Fig. 5 illustrates our results on rain detection and removal.

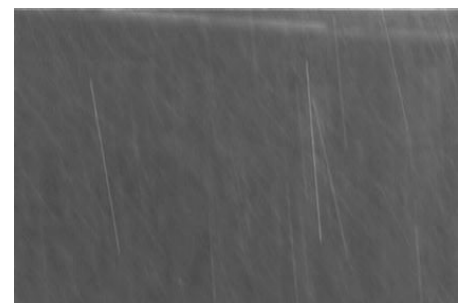

(a) An Original frame with rain in video

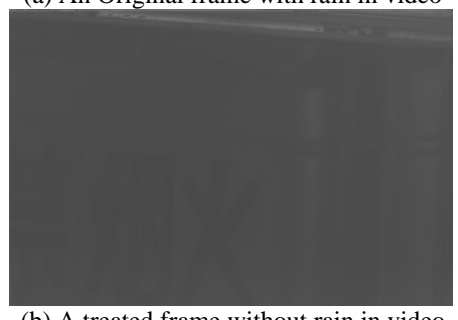

(b) A treated frame without rain in video

Fig. 5: Rain detection and removal of similar neighboring area in video

\section{Conclusion}

In order to detect rain in video, improved histogram models containing temporal and spatial properties for stationary and dynamic scenes are established. K-Means Clustering is applied to obtain the centers of background and rain; moreover, a parameterized method is introduced in. Further research will be on real-time treatment using physical directional property and background segmentation, considering the misinformation of winged insects from motion-blurred rain and low-time processing.

\section{Acknowledgements}

This work was supported by National Natural Science Foundation of China(607 02032), and Natural Scientific Research Innovation Foundation in Harbin Institute of Technology(HIT.NSRIF.2008.63)

\section{References}

[1] K. Garg and S.K. Nayar, "Detection and removal of rain from videos," in Proc. CVPR 2004, vol. 1, pp. 528535.

[2] S.G.Narasimhan and S.K.Nayar, "Vision and the atmosphere,” IJCV2002, vol.48, no.3, pp.233-254.

[3] Bertalmio M, Vese L, Sapiro G, et al, "Simultaneous structure and texture image inpainting," IEEE Transactions on Image Processing 2003, vol.12, no.8, pp.882- 889

[4] K.Garg and S.K. Nayar, "Vision and Rain,” IJCV2007, vol.75, no.1, pp.327.

[5] http://www.math.ucla.edu/ imagers/h tmls/inp.html.

[6] P.Barnum, T.Kanade and S.G. Narasimhan, "Spatio-temporal frequency analysis for removing rain and snow from videos," in Proc. ICCV2007, workshop PACV

[7] X.Zhang, H.Li, Y.Qi, et al, "Rain removal in video by combining temporal and chromatic properties," in ICME2006.

[8] R.M Manning, "Stochastic electromagnetic image propogation,” McGraw-Hill, Inc, 1993.

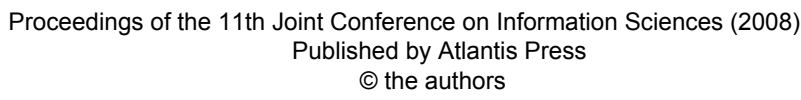

